\title{
Is chronic detergent ingestion harmful to the gut?
}

\author{
L A MERCURIUS-TAYLOR, A P JAYARAJ, AND C G CLARK \\ From the Surgical Unit, School of Medicine, University College, London, The Rayne Institute London WC1E \\ 6JJ, UK
}

ABSTRACT Synthetic detergents are used in large quantities as household and industrial cleaners. Because of the common practice of leaving dishes washed in detergent solutions to dry without rinsing these compounds are ingested. We have calculated that an adult takes in about $1 \mathrm{mg} / \mathrm{kg}$ detergent a day and babies can be administered between seven and $10 \mathrm{mg} / \mathrm{kg}$ a day. Rats were fed a dose of $100 \mathrm{mg} / \mathrm{kg}$ a day in a pilot experiment and gross abnormalities were found in the gastrointestinal tract, the most striking being subtotal villous atrophy of the small bowel mucosa and glandular atrophy in the colon. These changes were not reversible 12 weeks after cessation of detergent administration.

The development of synthetic detergents 30 years ago revolutionised cleaning processes. These comparatively cheap materials are used in the manufacture of washing up liquids, bath oils and foams, shampoos, soap powders, and numerous other household and industrial cleaners. It is common practice to leave glasses, crockery, and eating implements to dry with a coating of detergent, and indeed some manufacturers recommend this since the fluorescent additives cause them to "sparkle." These compounds are inevitably ingested from such implements. Feeding bottles for babies are often steeped in strong solutions of detergent and not rinsed between feeds.

Detergents have a potent effect on cell membranes. At low concentration they form complexes with brush border enzymes ${ }^{1}$ and at higher concentrations solubilise membranes by forming micelles with the lipid protein components. ${ }^{23}$ They have provided membrane biologists with a useful tool for the study of membrane components in vitro. What are the effects of these substances in vivo? We present a descriptive account of the effects of chronic ingestion of synthetic detergent on the morphology of the gastrointestinal tract of the rat.

\section{Materials and methods}

Six female Wistar rats, litter mates of average weight $200 \mathrm{~g}$, were caged together and fed a standard 41B diet. The drinking water was a $1 \%$ solution of a househould detergent (Fairy Liquid: Proctor \&

Received 6 December 1982

Accepted 14 March 1983
Gamble $37 / 8077 / G$ ) in tap water.

The animals were weighed regularly and the faeces inspected daily. The amount of solution consumed each day was noted. Single, randomly chosen rats were killed at regular intervals between 18 and 68 weeks by ether inhalation in a closed jar, and postmortem examination was performed immediately. After inspection of the viscera in situ, a $6 \mathrm{Ch}$ polyethylene cannula was passed into the stomach through the mouth and the entire gastrointestinal tract lavaged gently with physiological saline, thus clearing it of intestinal contents. The bowel was then filled with $10 \%$ buffered formol saline. Segments of intestine were then isolated and tied so as to retain intraluminal formalin and to ensure immediate mucosal fixation. These segments comprised oesophagus to duodenojejunal junction, small bowel to just proximal to the ileocaecal junction, and finally caecum to rectum. They were placed in $10 \%$ buffered formol saline for external fixation. All accessory organs of digestion were excised and fixed for 24 hours.

After fixation, the gastrointestinal segments were cut open along the antimesenteric border and the mucosa inspected. Segments were then made into a "swiss roll" and processed for histological examination. This technique allows microscopic examination of the mucosa of the entire gastrointestinal tract. Processed tissues were embedded in wax and cut at $4 u$ on a Leitz rotary microtome before staining with haemotoxylin and eosin, PAS, and alcian blue.

Fairy Liquid consists of $40 \% \mathrm{w} / \mathrm{v}$ anionic alkyl benzene sulphonate, approximately $60 \% \mathrm{w} / \mathrm{v}$ nonionic alkyl ether sulphate, and a small amount of blue-green colour agent. One of the components of 
this detergent possesses an absorption peak at 235 $\mathrm{mm}$ and was used to quantify the amount of detergent remaining on an unrinsed standard $23 \mathrm{~cm}$ diameter dinner plate, a $230 \mathrm{ml}$ glass tumbler, and a baby's feeding bottle. A washing solution of $5 \mathrm{ml}$ detergent in 21 of tap water was made (this is probable less than the concentration a housewife would use for dishwashing). Dilutions of this solution were read in a Unicam SP 1700/1800 ultraviolet spectrophotometer (Pye Unicam Ltd) at $235 \mathrm{~mm}$, using tap water as a zero reference, and optical density was plotted against concentration. The utensils were washed in the fluid and allowed to dry. The receptacle surface of each utensil was washed with $20 \mathrm{ml}$ of water and this fluid read in the spectrophotometer. An additional quantification was made for the baby's feeding bottle after steeping in a strong solution of detergent solution $(10 \mathrm{ml} / \mathrm{l})$.

\section{Results}

The dilution curve of optical density against concentration obeyed Beer's Law and gave a straight line through the axis. From this plot the concentration of detergent remaining on the utensils was calculated, assuming even dispersion of fluorescent detergent molecules in solution. For three meals, six cups of coffee, tea, or water, and a glass of beer the amount of detergent ingested a day was calculated at $75 \mathrm{mg}$. Calculation for a standard feeding bottle at two detergent concentrations showed that a baby can be administered dosages of anything between 150 and $250 \mathrm{mg}$ of detergent daily. Each rat consumed $35 \mathrm{mg}$ detergent a day.

The animals gained weight steadily. The growth curves of the detergent fed rats were compared with a mean growth curve of 10 control female Wistar rats of initially similar age and weight over the same period. There was little difference in the rate of weight gain over the period of growth, but the maximum attained weight of the test rats was below that of the controls. There were no external signs of illness, and none of the animals developed diarrhoea.

At necropsy the bowel wall of all the rats was noted to be thinner and more transparent than normal. This became more obvious during luminal lavage.

\section{Histology}

The observed pathological changes although remarkably constant in all the rats, tended to be more florid in those killed later.

Tongue-There was gross hyperkeratinisation of the surface of the tongue with total disappearance of the taste buds in all rats.
Oesophagus - Large areas of the oesophagus were denuded of mucosa but where present, this was thin and friable with an uneven, frayed, and easily detachable keratin layer. This attenuated mucosa contained only a single layer of basal cells compared with three or more in the normal rat oesophagus. No strictures were seen, and there was little inflammatory reaction in the ulcerated areas, but the features were compatible with corrosive oesophagitis.

Stomach-The stomach appeared histologically normal in all specimens examined.

Small intestine-Grossly abnormal changes were seen throughout the small bowel. Villi were considerably reduced both in number and size. They were oedematous and friable and few were frankly necrotic. Extensive atrophy of the mucosal glands was evident through cell hyperplasia. These changes were progressively more noticeable in the distal part of the small bowel. Mitotic figures were so few as to suggest a failure of normal regenerative activity. There was slight cellular infiltration in the lamina propria. Submucosal lymphoid aggregates were more prominent in rats killed at an early stage but particularly sparse in those killed later (fig 1).

Large intestine - There was generalised glandular atrophy and glands were small but widely separated in the stroma. The swollen and distorted columnar epithelial cells were seen sloughed off from the mucosa (we are sure this is not autolytic surface loss because of the method of preparation described

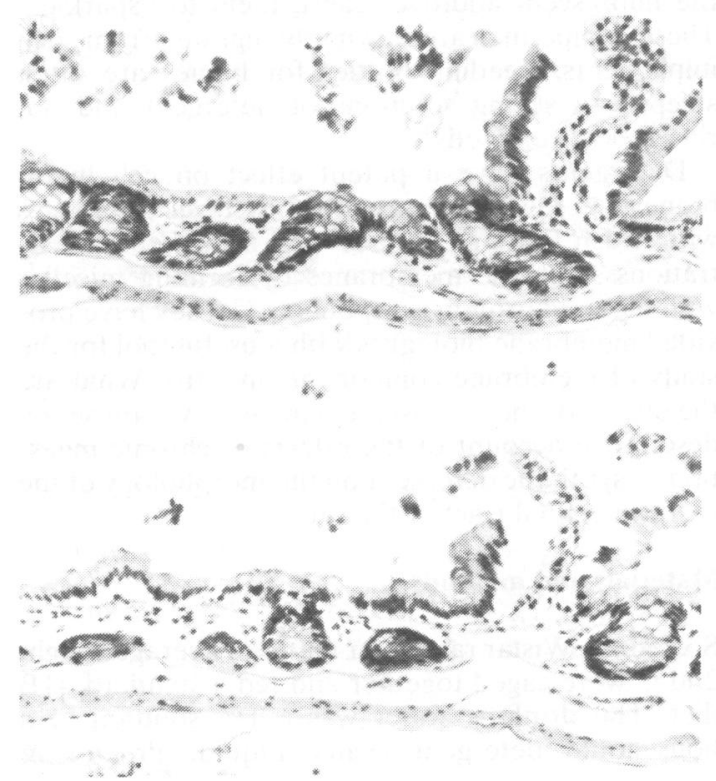

Fig 1 Longitudinal section of part of a roll of jejunum showing sparse stunted villi and glands and numerous goblet cells. (Haematoxylin and eosin $\times 100$ ) 


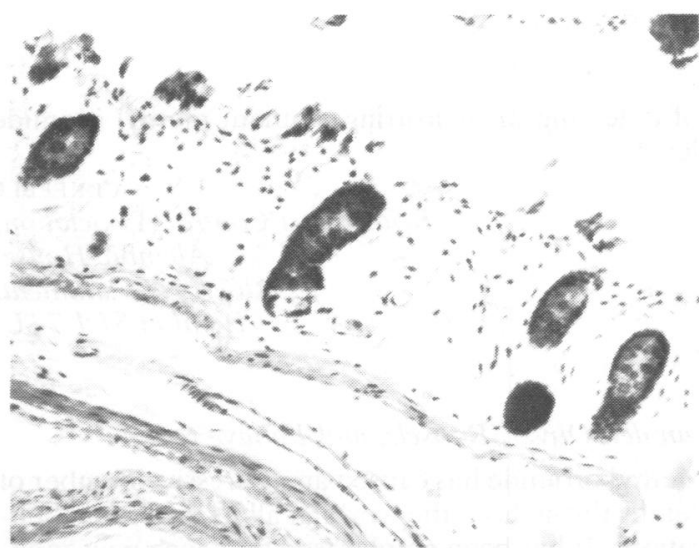

Fig 2 Longitudinal section of part of a roll of colon showing subtotal glandular atrophy. (PAS and Alcian blue $\times 125$ )

above). The slight cellular infiltration seen was confined to mucosa and submucosa without penetrating the muscularis (fig 2).

Liver-The liver showed an almost normal histological picture with mild periportal fatty infiltration.

Pancreas-Sections of the pancreas were difficult to cut because of microcalcification. These deposits tore gaps in the tissue and blunted the knife. Efforts to show this calcification radiologically in the anaesthetised rat and later in the pancreatic tissue itself, using low kilovoltage mammographic equipment, were not convincing, presumably because of the size of these deposits. The remaining pancreatic parenchyma appeared normal.

\section{Discussion}

There have been reports of corrosive oesophagitis after drinking dishwater detergent $t^{4}$ and acute colitis after administration of detergent enemas. ${ }^{5}$ The use of gastrograffin enemas in the treatment of meconium ileus ${ }^{6}$ resulted in several cases of fulminating enterocolitis with delayed perforation of the bowel, for which there was no rational explana- tion at the time. Gastrograffin contains the synthetic detergent Tween 80 . The effect of detergent ingestion on lipid absorption has been reported, both after a single dose of hydrophobic detergent (138 $\mathrm{g}$ of Plurome L-81) in rats and after chronic feeding of a $1 \%$ solution of this detergent, over seven weeks. ${ }^{7}$ Abnormalities of fat absorption with steatorrhoea were described in these experiments.

We know that manufacturers toxicity test their products to a $1 \%$ concentration, but we are not aware of any published reports of their findings. We have shown profound morphological changes in the gastrointestinal tract in all our rats with this concentration.

Clearly, it would be inappropriate to extrapolate these results to man, particularly after a pilot experiment where our animals received dosages of, on average, $100 \mathrm{mg} / \mathrm{kg}$ a day compared with $1 \mathrm{mg} / \mathrm{kg}$ a day for an adult. Nevertheless, we would like to draw attention to the potential dosages of between 35 and $50 \mathrm{mg}$ a day in babies weighing about $5 \mathrm{~kg}$ and think it possible that early mucosal damage may lead to chronic bowel disease.

Detergents are a useful if not indispensable part of modern living, but we should take precautions against eating them.

\section{References}

' Colbean A, Maroux S. Integration of alkaline phosphatase in the intestinal brush border membrane. Biochem Biophys 1978;511:39-51.

${ }^{2}$ Baugham JA, Lea EJA. The interaction of detergents with bilayer lipid membranes. Biochem Biophys Acta 1978;511:388-96.

${ }^{3}$ Helenius A, Simons K. Solubilization of membranes by detergents. Biochem Biophys Acta 1975;415:29-79.

4 Jeppsson P-H, Ejnell H. Mucous membrane damage infected by dishwater detergent. Lakantidningen 1980;77:642-4.

5 Kirchner SG, Buckspan GS, O'Neill JA, Page DL, Bunko H. Detergent enema: a cause of caustic colitis. Paed Radiol 1977;6:141-6.

- Noblett HR. Treatment of uncomplicated meconium nens by Gastrografin enema: preliminary report. J Paed Surg 1969;4:190-7.

${ }^{7}$ Brunette CW, Bochenek WJ, Abraham R, Rodgers JB. Effect of hydrophobic detergent on lipid absorption in the rat and on lipid and sterol balance in the swine. Dig Dis Sci 1979;24:718-25. 